\title{
ZU SPRACHE UND DATIERUNG DES AMDUAT
}

\author{
By KARL JANSEN-WINKELN
}

In recent years it has often been maintained that the texts of the 'Amduat' should date to the Old Kingdom because they contain so many linguistic features known from Old Egyptian. On closer inspection this view cannot be corroborated. It is true that there are many words and forms that look very ancient, and some of them may indeed have their origin in Old Egyptian texts. But most of these elements are different in certain aspects (writing, syntax, or frequency) from those found in genuine texts of the Old Kingdom. The more probable conclusion is that most of them have been adopted to make the texts of the Amduat look as if they had been composed in very ancient times.

DAs früheste Exemplar des Unterweltbuches, das unter dem Namen 'Amduat' bekannt ist, ${ }^{\mathrm{I}}$ stammt aus dem Begräbnis Thutmosis' I. oder der Hatschepsut. ${ }^{2}$ In welcher Zeit der Text aber verfasst wurde, ist seit langer Zeit umstritten, die Vorschläge reichen von der frühen I 8. Dynastie über das Mittlere Reich und die Erste Zwischenzeit bis hinauf zum Alten Reich.

E. Hornung, die erste Autorität auf dem Gebiet der Unterweltsbücher und Herausgeber der maßgeblichen Editionen des Amduat, ${ }^{3}$ hat sich aufgrund sprachlicher und mehr noch inhaltlicher Indizien immer für eine Datierung in den Beginn der I 8 . Dynastie oder wenig früher ausgesprochen, während er den Einfluss älterer Motive einräumt. ${ }^{4}$ Einen ähnlichen Ansatz vertraten auch S. Schott ${ }^{5}$ und H. Brunner. ${ }^{6}$ Daneben sind aber schon seit längerer Zeit andere Meinungen vertreten worden, die die Entstehung des Amduat in viel frühere Zeit setzen. Schon H. Grapow dachte an eine Redaktionsgeschichte, die weit vor das Neue Reich zurückreicht, ${ }^{7} \mathrm{H}$. Altenmüller datierte das Amduat ins Mittlere ${ }^{8}$ oder sogar ins Alte Reich, ${ }^{9}$ E. Wente ins Mittlere

' Zur eigentlichen Bedeutung der Bezeichnung 'Amduat', s. E. Hornung, Die Grabkammer des Vezirs User (NAWG 1961/5; Göttingen, 1961), 103-4; S. Schott, Die Schrift der verborgenen Kammer in Königsgräbern der I 8. Dynastie (NAWG 1958/4; Göttingen, r 958 ), 334.

2 S. jetzt F. Mauric-Barberio, 'Le premier exemplaire du Livre de l'Amdouat', BIFAO Ior (200I), 31 5-50.

3 E. Hornung, Das Amduat: Die Schrift des verborgenen Raumes, I-III (ÄA 7 and I3; Wiesbaden, I967) (im folgenden $A m d$.); id., Texte zum Amduat, I-III (AH I3 $^{-1} 5$; Geneva, 1987-1994) (im folgenden: $T z A$ ).

4 Hornung, Die Grabkammer des Vezirs User, ro4; Amd. I, xi; III, 56-7; id., Ägyptische Unterweltsbücher (Darmstadt, 1984), I7-18; id., Altägyptische Fenseitsbücher: Ein einführender Überblick (Darmstadt, 1997), 40. Auch A. Piankoff, der sich als erster intensiv mit den Unterweltsbüchern beschäftigte hatte, datierte das Pfortenbuch ins Neue Reich, s. C. Maystre und A. Piankoff, Le livre des portes, I (MIFAO 74; Kairo, I939), x.

5 Schott, Die Schrift der verborgenen Kammer, 332-3.

${ }^{6}$ H. Brunner, Grundzüge der altägyptischen Religion (Darmstadt, I983), 137. Vgl. aus jüngerer Zeit auch H. Schlögl, Der Gott Tatenen (OBO 29; Freiburg und Göttingen, I980), 2 I und E. Hermsen, Die zwei Wege des fenseits (OBO I I 2; Freiburg und Göttingen, I992), 35 n. I4 und 41 n. 35.

7 H. Grapow, 'Studien zu den thebanischen Königsgräbern', ZÄS 72 (г936), 32-6.

${ }^{8}$ H. Altenmüller, 'Zur Überlieferung des Amduat', $\mathscr{F E O L ~} 20$ (1967-8), 42.

9 H. Altenmüller, in B. Spuler (ed.), Handbuch der Orientalistik, I: Ägyptologie, II. Abschnitt: Literatur (Leiden, 1970), 72: 'vielleicht bis ins Alte Reich (4./5. Dyn.?)'. 
Reich oder die I. Zwischenzeit, ${ }^{10}$ ähnlich U. Rößler-Köhler, ${ }^{11}$ J. Assmann, ${ }^{12}$ J. Baines, ${ }^{13}$ und neuerdings J. Wegner und J. Roberson. ${ }^{\mathrm{I}}{ }^{2}$ Einen ausführlichen Überblick über die verschiedenen älteren und jüngeren Meinungen hat zuletzt S. Wiebach-Koepke gegeben, ${ }^{15}$ die sich selbst aufgrund inhaltlicher Erwägungen (Konzept eines dreiteiligen Weltbilds mit konsequenter Abgrenzung von Himmel, Erde, und Unterwelt) für eine 'junge' Datierung in die 2. Zwischenzeit oder das frühe Neue Reich ausspricht. ${ }^{16}$

Die Sache wird nicht einfacher dadurch, dass für die Frage nach der Datierung mehrere Ebenen in Betracht kommen: ${ }^{17}$

a) Die Gattung Unterweltsbücher insgesamt. Das Amduat ist zwar das am frühesten belegte Exemplar dieser Gattung, aber darum noch nicht zwingend das älteste (als das es aber wohl von den meisten angesehen wird). In jedem Fall haben die Unterweltsbücher und die verwandten Texte (wie die Sonnenlitanei) inhaltlich, sprachlich, und pragmatisch viele Gemeinsamkeiten. Mögliche Datierungskriterien für jedes einzelne dieser Bücher müssen auch für die anderen in Betracht gezogen werden.

b) Das (in sich abgeschlossene) Buch 'Amduat', so wie es in den Gräbern der I 8 . Dynastie vorliegt. ${ }^{18}$

c) Die Quellen für dieses Werk (bzw. allgemein für die einzelnen Unterweltsbücher). Das Amduat ist kaum wie ein literarisches Werk in einem Zug geschaffen worden. Es ist nicht nur in zwölf Einzelstunden gegliedert, es gibt auch innerhalb dieser Stunden klar erkennbar unterschiedliche Bestandteile: deskriptive Teile, Vermerke über Nützlichkeit, Anbringung etc., Wechselreden (nur in Std.I-3) u.a.m. Das könnte unter Umständen bedeuten, dass einzelne Teile des Buches unterschiedlichen Datums sind. ${ }^{19}$

Im folgenden geht es in erster Linie um die Datierung der Gesamtkomposition, also um b).

Für eine solche Datierung der Gesamtkomposition ist zunächst die Frage von Bedeutung, ob das Amduat von Anfang an für den toten König bestimmt und für die Königsgräber des Neuen Reiches konzipiert war oder nicht. Falls ja, sollte es auch-

${ }^{10}$ E. Wente, 'Mysticism in Pharaonic Egypt', FNES 4r (r982), I 76.

II U. Rößler-Köhler, 'Ein Gottesbegräbnis des Mittleren Reiches in königlichem Kontext: Amduat, 4. und 5. Stunde', in R. Gundlach und W. Seipel (eds), Das frühe ägyptische Königtum (ÄAT 36.2; Wiesbaden, I999), 73-96 (spätestens ins Mittlere Reich).

${ }_{12}$ J. Assmann, Der König als Sonnenpriester (ADAIK 7; Glückstadt, I970), 57 nn. 2-3; id., Re und Amun (OBO 5I; Freiburg und Göttingen, I983), I3 n. 25.

${ }_{3} \mathrm{~J}$. Baines, 'Interpreting the Story of the Shipwrecked Sailor', $\mathscr{F E A} 76$ ( 1990$), 63$.

${ }^{14}$ J. Wegner, 'The Tomb of Senwosret III at Abydos: Considerations on the Origins and Development of the Royal Amduat Tomb', in D. Silverman, W. K. Simpson, und J. Wegner (eds), Archaism and Innovation: Studies in the Culture of Middle Kingdom Egypt (New Haven, 2009), 146-50; J. Roberson, "The Early History of "New Kingdom” Netherworld Iconography: A Late Middle Kingdom Apotropaic Wand Reconsidered', in Silverman et al. (eds), Archaism and Innovation, 427-45.

${ }_{15}$ S. Wiebach-Koepke, Phänomenologie der Bewegungsabläufe im fenseitskonzept der Unterweltsbücher Amduat und Pfortenbuch und der liturgischen 'Sonnenlitanei' (ÄAT 55; Wiesbaden, 2003), 23-6.

${ }^{16}$ Ibid., 26-9.

${ }_{17}$ Vgl. dazu für die Unterweltsbücher insgesamt auch M. Müller-Roth, Das Buch vom Tage (OBO 236; Freiburg und Göttingen, 2008), 54I-4.

${ }^{18}$ In den Königsgräbern der Ramessidenzeit ist es bekanntlich nicht als geschlossene Komposition überliefert, sondern auf bestimmte Stellen im Grab verteilt, oft nur Auszüge des Buches. Die Verteilung im einzelnen läßt sich den Publikationen Hornungs entnehmen, die Gründe dafür sind unbekannt und noch nicht wirklich untersucht worden.

19 Vgl. Grapow, $Z \ddot{A} S$ 72, 32-5. Die Stunden 4 und 5 sind öfter als deutlich älter eingeschätzt worden: ibid., 34-5; vgl. auch Rößler-Köhler, in Gundlach und Seipel (eds), Das frühe ägyptische Königtum, 73-96. 
zumindest in der vorliegenden Form-zu Beginn des Neuen Reiches entstanden sein. Es scheint aber einiges darauf zu deuten, dass diese Texte ursprünglich nicht als Wanddekoration von Königsgräbern konzipiert waren:

a) Der König kommt nicht darin vor, außer in sekundären Einschüben. ${ }^{20}$

b) Die Verteilung auf die Wände der Gräber entspricht in keinem einzigen Fall den Anweisungen im Text; ${ }^{21}$ die ovalen Sargkammern der Königsgräber der frühen I8. Dynastie haben nicht einmal vier Seiten.

c) In den Gräbern von Thutmosis I. und III. ist ein Papyrus abgebildet, im Grab Thutmosis' I. sogar im Originalformat. ${ }^{22}$ Es handelt sich also ersichtlich um die 'sekundäre Verwendung' eines Textes, und noch deutlicher wird das dadurch, dass man einen alten und schon lückenhaften Papyrus als Vorlage genommen hat. ${ }^{23}$ Wäre das Amduat von Anbeginn für die Dekoration der Königsgräber bestimmt gewesen, wäre es auch als Wanddekoration konzipiert worden.

d) Der Text ist-zumindest in seinen wesentlichen Teilen-ganz überwiegend deskriptiv: Orte, Wege, Jenseitswesen, und ihre Tätigkeiten werden stichwortartig beschrieben. Für funeräre Inschriften, die im allgemeinen aus wörtlichen Reden bestehen, wäre das sehr ungewöhnlich.

Wenn dieses 'Buch' aber ursprünglich einen anderen 'Sitz im Leben' hatte, ist seine Datierung wieder völlig offen, und man muss nach anderen Möglichkeiten suchen, sie näher einzugrenzen.

In jüngerer Zeit haben vor allem J. F. Quack und A. von Lieven darauf gedrängt, bei der Datierung von Texten verstärkt sprachliche Kriterien heranzuziehen. ${ }^{24}$ Tatsächlich muss man feststellen, dass bei den meisten der oben zitierten Datierungsvorschläge sprachliche Kriterien - wenn überhaupt-nur eine untergeordnete Rolle gespielt haben, obwohl das doch der nächstliegende Anhaltspunkt sein sollte. Quack hat verschiedentlich klargemacht, dass er die Sprache der Unterweltsbücher für altägyptisch (oder zumindest für sehr stark altägyptisch beeinflusst) hält, und vertritt daher mit Nachdruck die Überzeugung, dass sie im Alten Reich entstanden sind. ${ }^{25}$

In der Tat ist es offenkundig, dass die Texte des Amduat eine ganze Reihe von Schreibungen, Formen, und Konstruktionen enthalten, wie sie für die Sprache des Alten Reiches charakteristisch sind oder doch zu sein scheinen. Dennoch ist, wie sich aus dem Folgenden ergeben wird, die Sprache des Amduat keineswegs insgesamt

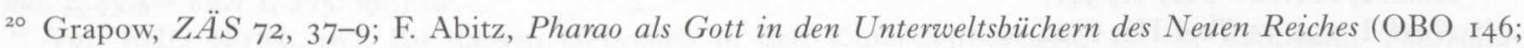
Freiburg und Göttingen, 1995), 23-6.

${ }^{21}$ Schott, Die Schrift der verborgenen Kammer, 332-3. Seine Vermutung, das Amduat sei für das-bislang nicht sicher identifizierte-Grab Amenophis' I. konzipiert, ist reine Spekulation.

${ }^{22} T z A$ I, p. x (I).

${ }^{23}$ Wente, fNES $_{4} \mathrm{I}, \mathrm{I} 64$.

${ }^{24}$ A. von Lieven, Grundriss des Laufes der Sterne (CNI 31; Kopenhagen, 2007), 223-57; J. Quack, 'Corpus oder membra disiecta', in W. Waitkus (ed.), Diener des Horus: Festschrift für Dieter Kurth zum 65. Geburtstag (Hamburg, 2008), 208-28.

${ }_{25}$ Zur Datierung des Pfortenbuchs ins Alte Reich, s. J. Quack, Rezension von J. Zeidler, Pfortenbuchstudien (Wiesbaden, 1999), BiOr 57 (2000), 547-58, bes. 552; zur Datierung aller Unterweltsbücher (außer dem Höhlenbuch) ins Alte Reich, s. ibid., 558; zur Datierung des Höhlenbuchs, ibid., 549 n. 15; zur Datierung der Sonnenlitanei, ibid., 559 (Anhang); zur Datierung des Buchs von der Erde und von 'TB I68', ibid., 558 n. 39 ; zur Datierung des Buchs von der Nacht ins Alte Reich, s. J. Quack, Rezension von G. Roulin, Le livre de la nuit (Freiburg und Göttingen, I996), WdO 28 ( 1997), I 77-8 I, v.a. I79-80. Auch nach A. von Lieven ist das Amduat sprachlich klar altägyptisch, s. Grundriss des Laufes der Sterne, 2 Io, n. I I45: 'der eindeutig altägyptische Befund für das Amduat ... '; ebenso die meisten anderen Unterweltsbücher und vergleichbare Texte: ibid., 278: '... als Altägyptisch erwiesen' (!). 
altägyptisch, der Befund ist tatsächlich recht widersprüchlich. An distinktiven Merkmalen zur Bestimmung der Sprachstufe lässt sich (u.a.) ${ }^{26}$ folgendes anführen:

\section{A) Orthographisches}

Es kommen einige deutlich altägyptische Schreibungen vor, z.B. $\lambda^{*}(=j w . f),{ }^{27} \AA$ für $j m j$ 'gib', ${ }^{28}$ oder $\Rightarrow$ als Infinitiv von $w d j .{ }^{29}$ Dies könnte auch für Wörter gelten wie

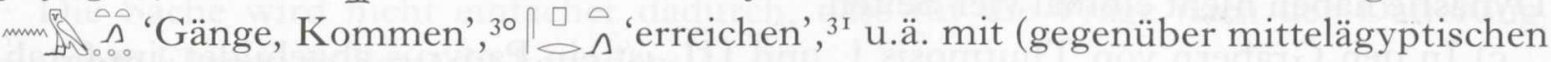
'Standard') zusätzlichen Einkonsonantenzeichen. ${ }^{32}$

Vergleichbare Schreibungen scheinen im Amduat allerdings manchmal etwas übertrieben oder ungewöhnlich und daher suspekt, z.B. ㄴm If 'Zugehörige', ${ }^{33}$

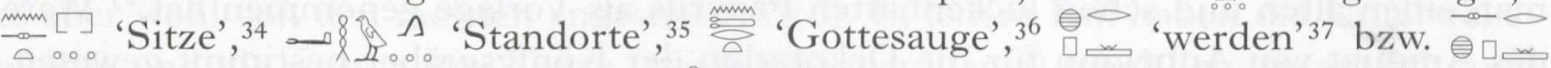

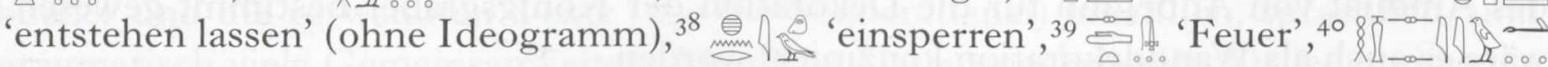
'singende', ${ }^{41}$ usw., wobei die letzten Beispiele auch lautlich eine 'jüngere' Gestalt haben. Man könnte daher zunächst annehmen, ein alter Text werde von einem Schreiber in zeitgenössischer Orthographie (der r 8. Dyn.) wiedergegeben. Aber Schreibungen wie $\stackrel{-\infty}{\leftrightarrows}$ 'Feuer' 42 oder $\stackrel{-\infty}{\leftrightharpoons}$ 'speisen' ${ }^{-\infty}$ sind weder im Alten Reich noch sonst außerhalb der Unterweltsbücher geläufig. Und eine Form wie $\underset{\infty}{\infty}-\infty$ 'heilig', die für das gesamte Amduat (in allen Varianten!) ${ }^{44}$ geradezu typisch ist, ${ }^{45}$ kommt außerhalb der Unterweltsbücher m.W. überhaupt nicht vor. Dafür hat ganz gewiss kein alter Text als Vorlage gedient, und es kann auch nicht das Ergebnis einer 'Modernisierung' sein. Viel eher würde man an eine nicht ganz geglückte Nachahmung von Schreibweisen des Alten Reiches denken.

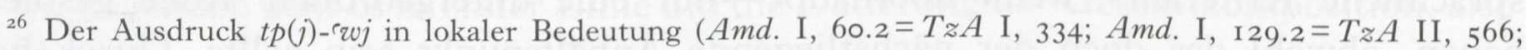
$A m d$. I, I98.10 $=T z A$ III, 822) ist kaum spezifisch altägyptisch, ebensowenig der Gebrauch der Partikel $j s$ im Nominalsatz nach Personalpronomen (Amd. I, I29.5=TzA II, 568). Auch das angebliche $j$-Augment in jhmjt 'Umstürzende' o.ä. (Amd. I, 77.I = TzA II, 392; vgl. Rößler-Köhler, in Gundlach und Seipel (eds), Das frühe ägyptische Königtum, 92 [8]) kann kaum herangezogen werden.

${ }_{27}$ Amd. I, 95.8=TzA II, 452; Amd. I, I24.4=TzA II, 548; vgl. E. Edel, Altägyptische Grammatik (AnOr 34/39; Rom, 1955/1964) (im folgenden: $\mathrm{E} A G$ ), §881.

${ }_{28}$ Amd. I, 77.4 = TzA II, 394; vgl. E $A G, \S 607$.

29 Amd. I, I 25.3 = TzA II, 552; vgl. E $A G, \S 693$.

3० $A m d$. I, 47.1 $=T z A$ I, 281 $; A m d$. I, r 86.3 = TzA III, 77I.

${ }^{31}$ Amd. I, 3.2 = TzA I, $\mathrm{I}_{3}$.

${ }^{32} \mathrm{E} A G, \S \S 27,52,58-9$; vgl. auch $\mathrm{I}_{6} 6$.

33 Amd. I, 121.10 = TzA II, 54I.

${ }_{34}$ Amd. I, 1 54.5 = TzA III, 652 .

35 Amd. I, I $28.7=T z A$ II, 564 .

${ }^{36}$ Amd. I, $103.7=T z A \mathrm{II}, 477$.

37 Amd. I, 108.6 = TzA II, 493.

${ }^{8}$ Amd. I, 53.I I $=T z A$ I, 305 .

39 Amd. I, 53.9 $=T z A$ I, 304 .

$4^{\circ} A m d$. I, 53.I I $=T z A \mathrm{I}, 305$.

${ }^{41}$ Amd. I, $13.12=T z A \mathrm{I}, 140$.

${ }^{42}$ Amd. I, 34.2 = TzA I, 210; $A m d$. I, 53.I I $=T z A \mathrm{I}, 305$.

${ }^{43}$ Amd. I, r69.5 = TzA III, 706 .

${ }_{44}$ Einzige Ausnahme ist $A m d$. I, I 2.3 $=T z A$ I, I36, wo zumindest eine Variante (User) die normale Schreibung hat.

${ }^{45}$ Amd. I, 42.5 = TzA I, 258; $A m d$. I, 57.3 = TzA I, 31 7; $A m d$. I, 62.5 $=T z A$ II, 344; $A m d$. I, 63.4 $=T z A$ II, 347; Amd. I, 67.2 = TzA II, 358; Amd. I, 67.4 = TzA II, 359; Amd. I, 67.9 $=T z A$ II, $36 \mathrm{r} ; A m d$. I, 68.4 $=T z A$ II, 362; $A m d$. I, 70.8 = TzA II, 369; $A m d$. I, 70.9 = TzA II, 370; $A m$ d. I, 71.2 = TzA II, 371; $A m d . \mathrm{I}, 76.4-5=T z A$ II, 389; Amd. I, 77.7 = TzA II, 395; Amd. I, 80. Iо =TzA II, 406; Amd. I, 8I.I = TzA II, 406; $A m d$. I, г I $8.4=T z A$ II, 527; $A m d$. I, г34.6 $=T z A$ II, $582 ; A m d$. I, г66.8 $=T z A$ III, 695; $A m d$. I, 174.5 $=T z A$ III, $724 ; A m d$. I, I $82.4=T z A$ III, $756 ; A m d$. I, I87.7 $=T z A$ III, 775 . 
Daneben finden sich auch einige 'Hyperkorrektheiten', die man bei einem wirklich alten Text nicht erwarten sollte, z.B. $\wedge$ (u.ä.) 'ablenken' (Kausativ von $t n m),{ }^{46}$ sowie nicht selten $\stackrel{\rightleftarrows}{\rightleftarrows}$ beim Demonstrativpronomen $t n$, manchmal sogar in allen Varianten. ${ }^{47}$ Und selbst die Femininendung wird zuweilen als $\approx$ notiert, z.B. $\frac{\pi}{\Delta}_{000}^{2}$ Wege'. ${ }^{4}$

\section{B) Demonstrativa}

Als Demonstrativa werden im Singular normalerweise $p n$ und $t n$ gebraucht (v.a. $n \underline{t} r$ $p n \varsigma_{3}$ ist sehr häufig), aber auch die älteren Formen $p w$ und $t w$ sind einigemal belegt. ${ }^{49}$ Auch Voranstellung der singularischen Pronomina ${ }^{5 \circ}$ findet sich: $p n$ sšmw 'dieses Bild', ${ }^{51} p n$ nt $r{ }^{3} 3$ 'dieser große Gott', ${ }^{2}$ ebenso selbständiger Gebrauch: $j p j j$ 'o jener' ${ }^{53}$ Aber diese Konstruktionen haben im Amduat (und das gilt auch für die anderen Unterweltsbücher) eine besondere Ausprägung, und es fragt sich, ob die wirklich altägyptisch ist. Im Alten Reich kommen selbständig gebrauchtes oder vorangestelltes $p n$ und $p f$ fast ausschließlich in paarweiser Gegenüberstellung vor:

PT I 42c: $m s j n . k p f j w r n . k p n$

'geboren ist dir jener, empfangen ist dir dieser'.

PT 425d: pf rw m- $\underline{h} n w$ pn rw

‘jener Löwe ist in diesem Löwen’.

PT 1376c/r377c: ... m pn gs rsj $n m r n h 3$... m pf gs $m h t j n m r n h 3$

'(binde die Stricke ...) auf dieser südlichen Seite des gewundenen Kanals ... (setze den NN auf deine Flügelspitze) auf jener nördlichen Seite des gewundenen Kanals'. ${ }^{54}$

Die Voranstellung im Alten Reich dient offenbar der besonderen Betonung; ${ }^{55}$ die Belege des Amduat (s.o.) sind dagegen offenbar bloße Varianten von sšmw pn bzw. nțr $p n$ '3. Und ein selbständiger Gebrauch im Vokativ wie $j p j j$ 'o jener' ist $\mathrm{m}$. W. im Alten Reich nie bezeugt.

${ }^{46}$ Amd. I, I I 7.6 = TzA II, 524 ( $5 \times$ sțnm; nur die beiden jüngsten Varianten haben stnm).

47 Z.B. Amd. I, $70.8=T z A$ II, 368 (7 $\times$ wst tn $n) ; A m d$. I, I I $7.8=T z A$ II, $525(6 \times n w t \underline{t} n)$.

${ }^{48}$ Amd. I, 69.I = TzA II, 364; vgl. auch $A m d$. I, 20.5 = TzA I, I62 (snsjwt).

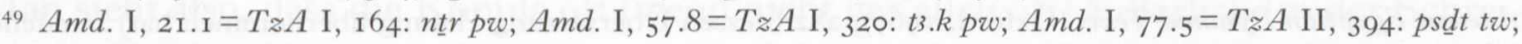
Amd. I, 45.5 = TzA I, 275: sht.f tw.

$5^{\circ} \mathrm{Vgl}$. E $A G \S \S_{1} 8_{5}$, I 87 . Zu diesem Gebrauch in den Unterweltbüchern, s.a. A. Baumann, The Suffix Conjugation of Early Egyptian as Evidenced in the Underworld Books (unveröffentlichte.PhD Diss., University of Chicago; Chicago, I998), 452 (13).

${ }^{51}$ Amd. I, 80.5 = TzA II, 404 .

${ }_{52}$ Amd. I, 159.3 = TzA III, 668; einziger Fall neben sehr häufigem ntrr 3 pn.

53 Amd. I, II9.9= TzA II, 533 .

54 Und daraus wird die Phrase $m$ pf gs $n m r n h 3$ mehrfach in Spr. 359 (PT 594b, d, e, f; 595b; 599d; 60ob) zitiert. Auch in den seltenen Fällen aus späterer Zeit treffen wir die Gegenüberstellung: Urk. IV, I19.I7; vgl. A. H. Gardiner, Egyptian Grammar (3. Aufl.; Oxford, I957), § I I I : rh.jpf3 rpn 'ich erkenne jenen (als unterschiedlich) von diesem'; auch Kairo CG 4224I, s. K. Jansen-Winkeln, Inschriften der Spätzeit, III: Die 25. Dynastie (Wiesbaden, 2009), 458: s'nh.n.k pfj mj pn 'du hast jenen belebt ebenso wie diesen'. In Fällen wie CT II, I6ra, jnk pn tn 'ich bin dieser und diese', könnte man sich fragen, ob hier nicht möglicherweise aus 'theologischen' Gründen an sich ungrammatische Formulierungen in Kauf genommen worden sind, indem die eigentliche Identifizierung (jnk $\mathrm{X} t n \mathrm{Y} p n)$ bewußt ausgelassen wurde. Jedenfalls dürften derartige Ausdrücke kaum als Datierungsmerkmale taugen.

${ }_{55}$ Vgl. dazu auch F. Hintze, “Konversion” und “analytische” Tendenz in der ägyptischen Sprachentwicklung', Zeitschrift für Phonetik und allgemeine Sprachwissenschaft 4 (1950), 4I-56; W. Schenkel, 'Die Konversion, ein Epiphänomen der kemischen (ägyptisch-koptischen) Sprachgeschichte’, $M D A I K$ 2I (I966), I23-32. 
Im Plural heißt es regelmäßig $n n(n)+$ Substantiv (im Plural) ${ }^{56}$ oder (seltener) $n w$ $n$ Substantiv (Pl.). ${ }^{57}$ Man könnte fast denken, dass Pluralformen wie $n w n$ Substantiv (P1.) hier die ältere Variante darstellen sollen, denn die eigentlichen altägyptischen Pluralformen $j p n / j p t n, j p w / j p t w$ etc. kommen im Amduat überhaupt nicht vor.

Die Pronomina der $n$-Reihe werden auch selbständig verwendet und haben dann z.T. pluralische Bedeutung: $j$ nw 'o jene'; ${ }^{8}$ jw jrj.tw nn $m$ rnw.sn 'man macht diese in ihren Namen'; ${ }^{59}$ nttnn nn jmjw nnw 'ihr seid diese, die im Nun sind'. ${ }^{\circ}$ Diese pluralische Bedeutung bei selbständigem Gebrauch der $n$-Reihe, die auch in anderen Unterweltbüchern und der Sonnenlitanei vorkommt, ${ }^{61}$ scheint erst seit der I 8 . Dynastie belegt zu sein. ${ }^{62}$

\section{C) Kopula}

Die Kopula wird häufig $p^{63}$ oder $p j^{64}$ geschrieben, aber daneben ist auch die Schreibung pw gängig. ${ }^{65}$ Dies entspricht grundsätzlich altägyptischem Gebrauch. ${ }^{66}$ Allerdings ist das Amduat in der Häufigkeit der Schreibungen $p$ oder $p j$ fast noch 'altägyptischer'

${ }^{5} n n(n) n t r w:$ Amd. I, 12.I $=T z A$ I, 135; $A m d . \mathrm{I}, \mathrm{1} 8.3=T z A$ I, I51; $A m d . \mathrm{I}, 25.8=T z A$ I, $184 ; A m d . \mathrm{I}, 97 \cdot 5=$ $T z A$ II, 457; $A m d$. I, 169.4=TzA III, 705; $A m d$. I, I94.4 $=T z A$ III, 804; nn (n) ntrvw ntryt: Amd. I, I3I.4 $=T z A$

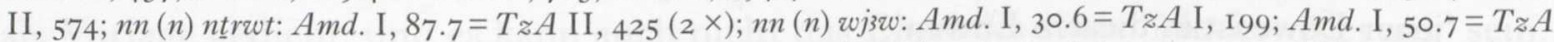
I, 293; Amd. I, 50.8 = TzA I, 294; nn (n) sšmw: Amd. I, 45.7 = TzA I, 276; Amd. I, I I3.I I = TzA II, 5I 3; nn n ḩwwt stst: $A m d$. I, I06.7 = TzA II, 487 .

57 nw $n$ sntj: $A m d$. I, 22.2 = TzA I, 170-1; nw n nțrwt: Amd. I, I 12.2=TzA II, 507; nww n ḩwwt štst: Amd. I, 106.5-6=TzA II, 487 (neben $n n n$ hrwwt, $A m d$. I, 106.7 $=T z A$ II, 487 ).

${ }^{8}$ Amd. I, $58.5=T z A$ I, 324 .

${ }_{59} A m d$. I, I $54 \cdot 3=T z A$ III, 650 .

${ }^{60}$ Amd. I, I77.5 = TzA III, 737-8.

6r Z.B. im Höhlenbuch: A. Piankoff, 'Le livre des Quererts', BIFAO 42 (1944), pl. xxx (links): jnk $n w R^{\circ}$ 'ich bin diese, (nämlich) Re' (vgl. E. Hornung, Ägyptische Unterweltsbücher, 345), oder häufig in der Phrase wnn nn $m$ shr $p n$ 'so sind diese beschaffen', s. Piankoff, BIFAO 42, pls xxxiii, xxxiv, xxxvi, xxxvii (vgl. Hornung, Ägyptische Unterweltsbücher, 35I, 352, 354, 355). In der Sonnenlitanei: z.B. E. Hornung, Buch der Anbetung des Re im Westen (Sonnenlitanei) (AH 2; Genf, r 975), 92: jnk w' $m$ nw jmjw-ht.k 'ich bin einer von diesen, die hinter dir sind'; ibid., 99-100: ... dww3.j nn $m$ 't jmnt 'damit ich diese im verborgenen Raum anbete'; ibid., I I 2-13: ... zšs $n n m \underline{d} d t . s n$ 'indem diese gemalt werden in ihrer Gestalt'.

${ }_{62}$ S. Wb. II, 273.I6. R. Hannig, Ägyptisches Wörterbuch, I (Mainz, 2003), 634c, führt einen angeblichen Beleg für $n n$ 'diese' aus den Pyramidentexten $($ I $950 \mathrm{e}=\mathrm{Spr}$. $667 \mathrm{~B})$ auf, aber auch dort ist gegen R. O. Faulkner, The Ancient Egyptian Pyramid Texts (Oxford, 1969), 282 (dessen Übersetzung Hannig übernommen hat) zweifellos 'dies' zu verstehen, wie auch J. Allen, The Ancient Egyptian Pyramid Texts (Atlanta, 2005), 326: im Kontext ist von anderen Personen überhaupt keine Rede.

${ }_{3}^{6} p$ : Amd. I, 79.8=TzA II, 403; Amd. I, 92.9=TzA II, 442 ( $\left.\times p j\right) ; A m d$. I, 94.7 = TzA II, 448; $A m d$. I, 94. Iо $=T z A$ II, 449; $A m d$. I, I26.I $=T z A$ II, 555; $A m d$. I, I26.I I $=T z A$ II, 558; $A m d$. I, г 8I.7 $=T z A$ III, 753; $A m d$. I, г81.8=TzA III, 753. Für $p$ mit-meist seltenerer-Variante $p w$, s: $A m d$. I, гоo.5=TzA II, $469 ; A m d$. I, $108.8=T z A$ II, 494; $A m d$. I, I I I.2 $=T z A$ II, 504; $A m d$. I, II 5.2 = TzA II, 51 7; $A m d$. I, I $6.2=T z A$ II, $52 \mathrm{I}$; $A m d$. I, г24.4 $=T z A$ II, 548; $A m d$. I, I28.6 $=T z A$ II, 563; $A m d$. I, I43.2 $=T z A$ II, 6 I I $A m d$. I, I44.9 $=T z A$ II, 6г7; $A m d$. I, г55.8=TzA III, 658; $A m d$. I, г57.6=TzA III, 663; $A m d$. I, г60.3 = TzA III, 672; $A m d$. I, I65.4 = TzA III, 690; Amd. I, 182.9 = TzA III, $75^{8}$.

${ }_{64}$ Amd. I, 74.4 = TzA II, 380 ; $A m d$. I, 82.7 $=T z A$ II, 4 I 2; $A m d$. I, 86.4 $=T z A$ II, $42 \mathrm{I} ; A m d$. I, 88.6 $=T z A$ II, 428; Amd. I, 89.7 = TzA II, 432; Amd. I, 92.2 =TzA II, 440; $A m d$. I, I I 8.7=TzA II, 529 .

${ }^{65}$ Amd. I, 9.4 = TzA I, 127; $A m d$. I, 9.5 = TzA I, 128; $A m d$. I, 22.7 $=T z A$ I, 172; $A m d$. I, 26.1 $=T z A$ I, 1 $85 ;$ Amd. I, 46.7 = TzA I, 281; Amd. I, 53.9 $=T z A$ I, 303; $A m d$. I, 64.5 $=T z A$ II, 35I; $A m d$. I, 69.3 = TzA II, $365 ;$ $A m d$. I, 93.1 = TzA II, 443; $A m d$. I, 105.2 = TzA II, 482; $A m d$. I, го6.7 $=T z A$ II, $487 ; A m d$. I, I 10.9=TzA II,

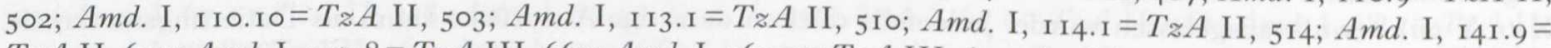
$T z A$ II, 607; $A m d$. I, г 57.8=TzA III, 664; $A m d$. I, I60. I $=T z A$ III, 67I; $A m d$. I, г70.7 $=T z A$ III, 71 I; $A m d$. I,

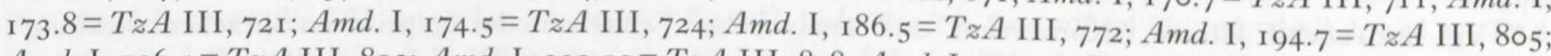
$A m d$. I, 196.4 = TzA III, 8I 2 ; $A m d$. I, 202.12 = TzA III, 838; $A m d$. I, 204.4 = TzA III, 845 .

${ }^{66} \mathrm{E} A G, \S \S \mathrm{I} 92-3$. 
als die Texte des Alten Reiches: dort findet man sie nur in der Pyramide des Unas häufiger. ${ }^{67}$

Bemerkenswert ist die Stellung der Kopula: im Altägyptischen steht sie nach Substantiv + attributivem Adjektiv (z.B. hrw nfr pw), im Mittelägyptischen zwischen Substantiv und Adjektiv (z.B. $t 3$ pw $n f r) .{ }^{68}$ Die Belege im Amduat zeigen alle die im Mittelägyptischen übliche Wortstellung zwischen Substantiv und attributivem Adjektiv:

wnwt pw tpt nt grh

'es ist die erste Stunde der Nacht'. ${ }^{69}$

sšm pj šts n jmht

'es ist das geheime Bild der $j m h t^{\prime} .^{70}$

sšm pw št $n$ dww

'es ist das geheime Bild der Unterwelt'. ${ }^{71}$

jrjw $p(w)$ št $t w n d w 3 t$

'es sind die geheimen Gestalten der Unterwelt'. ${ }^{72}$

š̌m pw šts $n H r(?)$

'es ist das geheime Bild des Horus'. ${ }^{73}$

Auch bei der Verbindung Substantiv + Partizip oder Relativform, wo im Altägyptischen sowohl die Stellung der Kopula nach als auch vor dem attributiven Verbaladjektiv belegt ist, ${ }^{74}$ kennt das Amduat ausschließlich diejenige Wortstellung, die auch die im Mittelägyptischen üblich ist, zwischen Bezugswort und Partizip:

hrw pw sdmw ntir pn

'eine Stimme ist es, die dieser Gott hört' ${ }^{75}$

$n t r j t p(w) n j k y t$ з $3 p p m$ dwst

'es sind die Göttinnen, die Apophis in der Unterwelt bestrafen' ${ }^{76}$

$n \underline{t} r w t$ pw zbjwt $m$ - ht $W$ sjr

'es sind die Göttinnen, die hinter Osiris gehen'. ${ }^{77}$

Man sieht also, dass die Kopula oft (wenn nicht gar allzu oft) äußerlich (in der Schreibung) der des Altägyptischen entspricht, ihre (weniger offensichtliche) syntaktische Verwendung aber mittelägyptischem Gebrauch.

${ }^{67}$ Vgl. E $A G, \S$ I 93. Da die Schreibung der Kopula als $p j$ im Amduat überwiegend (aber nicht ausschließlich) in den Stunden 4 und 5 vorkommt, wollte Grapow, $Z \ddot{A} S$ 72, 34, daraus schließen, dass diese Stunden älter sind als der Rest des Amduat. Die gleichfalls altertümliche Schreibung $p$ ist aber auch in den späteren Stunden reichlich belegt (s.o.).

${ }^{68} \mathrm{E} A G \S \$ 357,96 \mathrm{I}$.

${ }^{69} A m d$. I, 22.7 = TzA I, 172-3.

${ }^{70}$ Amd. I, 74.4 $=T z A$ II, $380-\mathrm{I}$.

${ }^{71}$ Amd. I, 105.2 = TzA II, 482 .

${ }_{72} A m d$. I, I 26. I I $=T z A$ II, $55^{8}$.

${ }^{73}$ Amd. I, $187.6=T z A$ III, 775.

${ }_{74} \mathrm{E} A G \$_{96 \mathrm{I}}$.

${ }^{75}$ Amd. I, $\mathrm{r} 06.7=T z A$ II, $487-8$.

${ }^{76} A m d$. I, 126.1 = TzA II, 555.

77 Amd. I, 1 57.8 $=T z A$ III, 664 . 


\section{D) Personalpronomina}

Als unabhängige Personalpronomina werden z.T. die alten Formen twwt und swt verwendet, ${ }^{78}$ daneben kommt aber auch $n t f$ vor. ${ }^{79}$ Ein solches Nebeneinander älterer und jüngerer Formen dieser Pronominalreihe gibt es schon im Alten Reich. Allerdings kommen die älteren Formen durchaus auch später noch vor, in den religiösen Texten des Mittleren Reiches und auch noch in der 18 . Dynastie. ${ }^{80}$

Das Objektpronomen st ist allerdings im Altägyptischen noch nicht belegt ${ }^{{ }^{1} \mathrm{r}} \mathrm{im}$ Amduat ist es aber recht häufig, meist $\left(\mathrm{I}_{7} \times\right.$ ) in den 'Nützlichkeitsvermerken'. ${ }^{82}$ Daraus könnte man zunächst schließen, dass diese 'Nachschriften' ${ }^{83}$ eben spätere Zusätze sind. Aber st kommt auch noch in anderen Kontexten vor; ${ }^{84}$ die 'Nützlichkeitsvermerke' sind wohl nur zufällig die Art von Text, wo ein Pronomen 'es' häufiger nötig ist.

Einigemal werden die 'enklitischen' Personalpronomina eben nicht enklitisch, sondern satzeinleitend verwendet, und zwar mit $\underline{t} n$ als Subjekt im Adverbialsatz:

$\underline{t} n$ m-hht.f sb3w.tn tpw-rwj.fj

'ihr seid hinter ihm, eure Sterne vor ihm'. ${ }^{85}$

$\underline{t} n$ r.t.tn $n . j s b 3 w . \underline{t} n n$ jmj $p t$

'ihr gehört zu mir, eure Sterne gehören zu dem im Himmel'. ${ }^{86}$

tn n $n . j m-h t . j$

'ihr gehört zu mir in meinem Gefolge' ${ }^{87}$

Ein weiterer möglicher Beleg ist unsicher, weil vor dem einleitenden $\underline{t} n$ eine freigelassene Stelle ist:

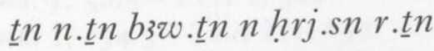

'euch gehören eure Seelen, sie haben sich nicht von euch entfernt'. ${ }^{88}$

Einmal findet sich auch einleitendes $s n$ vor einem Verbalsatz:

( $n \underline{t} r$ pn $\underline{d} w j$.f r.sn m-ht spr.f sn) sn $\underline{d} w j . s n$ n ntr pn $m$ hrw.sn

${ }^{78}$ twwt: $A m d$. I, 21.I = TzA I, 164; swt: $A m d . \mathrm{I}, 60.6=T z A$ I, 337; $A m d . \mathrm{I}, 86.9=T z A$ II, $423 ; A m d . \mathrm{I}, \mathrm{1} 29 \cdot 5=$ $T z A$ II, $568 ; A m d$. I, I43.6 = TzA II, 6I3. Dies kommt auch in den anderen Unterweltbüchern vor, s. Baumann, The Suffix Conjugation of Early Egyptian, 452 (I I).

79 Amd. I, 132.9 = TzA II, 578 .

8. R. van der Molen, A Hieroglyphic Dictionary of Egyptian Coffin Texts (PdÄ I 5 ; Leiden, 200o), 466-7, 753-4; Gardiner, $E G^{3}$, $\$ 64$ ('... have survived into Middle Egyptian as archaisms'); H. Brunner, Die Geburt des Gottkönigs (ÄA 10; Wiesbaden, 1964), I75 (mit Fußnote 3). Auch in der Berliner Lederhandschrift II.9, die ja schwerlich ins Alte Reich gehören kann, kommt twt 'du' vor: s. A. de Buck, 'The Building Inscription of the Berlin Leather Roll', in A. M. Blackman und H. Stock (eds), Studia Aegyptiaca, I (AnOr 17; Rom, 1938), 51.

8ı E $A G, \S \S$ 19, 169; C. Peust, 'Objektspronomina im Ägyptischen', LingAeg ro (2002), 310-1 7.

${ }^{82} A m d$. I, I 2.3 $=T z A$ I, 136; $A m d$. I, 34.5 = TzA I, 2 I I (U); $A m d$. I, 50.10 = TzA I, 295; $A m d$. I, 54.5 $=T z A$ I, 307; $A m d$. I, 63.7 = TzA II, 349; $A m d$. I, 76.9 =TzA II, 391; $A m d$. I, 99.3 =TzA II, $463 ; A m d$. I, I $8.10=T z A$ II, 530; $A m d$. I, г 23.4 = TzA II, 546; $A m d$. I, г25.6 = TzA II, 554; $A m d$. I, I 27.5 = TzA II, 560; $A m d$. I, 133.3 = $T z A$ II, 579; $A m d$. I, г35.6 = TzA II, 586; $A m d$. I, r64.I = TzA III, 686; $A m d$. I, г68.4 $=T z A$ III, 703; $A m d$. I, I 80.4 $=T z A$ III, 749; $A m d$. I, 193.7 $=T z A$ III, 799 .

$8_{3}$ S. Wiebach-Köpke, Sonnenlauf und kosmische Regeneration (ÄAT 71; Wiesbaden, 2007), 98-109.

${ }_{4}$ Amd. I, 102.5 = TzA II, 475; Amd. I, I46.6 = TzA II, 622 (vgl. Amd. I, I42.2 =TzA II, 609: hsp. hr sn); Amd. I, r63.I I $=T z A$ III, 685; $A m d$. I, 194.4 $=T z A$ III, 803-4.

${ }^{85}$ Amd. I, $129.2=T z A$ II, 566 .

${ }^{86}$ Amd. I, I 29.4 $=T z A$ II, 567 .

${ }^{87}$ Amd. I, I75.3 = TzA III, 728.

${ }^{88}$ Amd. I, 4I.2 = TzA I, 246. 
'(dieser Gott ruft ihnen zu, nachdem er sie erreicht hat), sie rufen diesem Gott zu mit ihrer Stimme'. ${ }^{89}$

Diese Konstruktion (in der Literatur z.T. sw s $\underline{d} m$.f genannt) ${ }^{9 \circ}$ ist in den anderen Unterweltsbüchern noch viel häufiger. Die Beispiele, die man dafür aus dem Alten Reich und den Sargtexten angeführt hat, sind nicht stichhaltig, ${ }^{91}$ die ersten sicheren Belege stammen aus der i 8. Dynastie, bemerkenswerterweise gerade aus solchen Texten, deren Datierung umstritten ist. Angesichts der doch sehr reichen Textüberlieferung aus dem Alten und Mittleren Reich mit so umfangreichen Textkorpora wie den Pyramidenund Sargtexten ist es daher kühn, darin einen archaischen Sprachgebrauch zu sehen; ${ }^{92}$ näherliegend ist ein (falscher) archaisierender.

Der Possessivausdruck $n . k-j m i j$ ist einmal in der 5 . Stunde bezeugt:

$n . k$-jm(jj) jmnt Re htp.k jm.s

'dir gehörig ist der Westen, Re, damit du in ihm ruhst'. ${ }^{93}$

Die Verbindung $n=+j m j j$ kennt man sonst erst ab dem Mittleren Reich. Der einzige altägyptische Beleg, der bisher dafür angeführt wurde, ${ }^{94}$ ist mehr als zweifelhaft. ${ }^{95}$

\section{E) Negationen und Negativsätze}

Wie im Altägyptischen scheint es auch im Amduat keine durchgehende graphische und funktionale Differenzierung zwischen so und $\mathrm{s}$ zu geben, letzteres kommt eigentlich nur als Variante vor. Das Fehlen der prädikativen Negation könnte allerdings zum Teil auch durch die Satzformen bedingt sein, die im Amduat gebraucht werden:

Negierte Adverbialsätze scheinen gar nicht vorzukommen und negative Existenzsätze nur in der merkwürdigen Verneinung zweier Infinitive ${ }^{96}(n m 33 n \text { ptr })^{97}$ bzw. durch jwtj adjektiviert. ${ }^{8}$

Die Negationen $n$ bzw. $n n$ sind daher fast nur vor finiten Verbalformen belegt, und zwar in den Konstruktionen $n s \underline{d} m . f$ (optativisch), ${ }^{99} n s \underline{d} m . f$ (präterital [?]) ${ }^{\text {Ioo }}$ und

\footnotetext{
${ }^{89}$ Amd. I, I46.4-5 = TzA II, 62 I-2. Eine weiterer entsprechender Beleg mit $\underline{t} n(A m d . \mathrm{I}, \mathrm{I} 9 \cdot 3=T z A$ I, I 57): $\underline{t} n$ $h p r . \underline{n} n$ Hprj ist wegen der Lücke vor $\underline{t} n$ ganz unsicher.

$9 \circ$ S. dazu generell H. Grapow, 'Zum Gebrauch der alten Pronomina absoluta', ZÄS 7I (I935), 48-55; W. Barta, 'Das Personalpronomen der $w j$-Reihe als Proklitikon im adverbiellen Nominalsatz', $Z \ddot{A} S$ I I 2 (I985), 94-ro4; m.E. nicht richtig gedeutet von J. Zeidler, Pfortenbuchstudien (GOF IV/36; Wiesbaden, I999), I, I 5 I-2 und 20I-4; s.a. K. Jansen-Winkeln, 'Sprachliche Bemerkungen zu den Unterweltsbüchern', $S A K{ }_{32}$ (2004), $219-23$.

${ }_{91}$ S. Quack, $\mathrm{BiOr}$ 57, 549 n. I3; W. Schenkel, 'Unterrichtsbehelfe und linguistische Theoriebildung in der Vermittlung der klassisch-ägyptischen Grammatik', $\mathrm{BiOr} 5^{8}$ (2001), 27; Jansen-Winkeln, $S A K$ 32, 220-1.

${ }_{92}$ So Quack, BiOr 57, 549 ('Reliktform und damit tendenziell alt'); vgl. auch von Lieven, Grundriss des Laufes der Sterne, 251-2; Brunner, Geburt des Gottkönigs, 173-5.

${ }_{93}$ Amd. I, 88. I = TzA, II, 427; vgl. dazu Jansen-Winkeln, $S A K$ 32, 230.

94 Urk. I, 77.r6, s. E $A G, \S_{3} 84$; H. Satzinger, 'Syntax der Präpositionsadjektive ("Präpositionsnisben”)', $Z A \ddot{S}$ I I3 ( I 986$)$, I 52 .

95 W. Schenkel, Memphis, Herakleopolis, Theben (ÄA I2; Wiesbaden, I965), 43, und neuerdings S. Grunert, 'Nur für Erwachsene: Political Correctness auf Altägyptisch?', $S A K_{37}$ (2008), 137-8. Zudem gehört die Inschrift wohl nicht mehr ins Alte Reich.

${ }_{96}^{6}$ Gardiner, $E G^{3}, 23 \mathrm{I}-2$.

97 Amd. I, 64.7-9=TzA II, 35I; Amd. I, 75.5-6=TzA II, 385; vgl. auch $A m d$. I, 76.7 =TzA II, 390.

${ }_{98}$ Amd. I, I I 8.5 = TzA II, 528 (jwtt mw.s jwtt sț.s); Amd. I, I 88.2=TzA III, 778 (jwtj rh.f); vgl. $S A K K_{32,} 212$.

${ }_{99}$ Amd. I, 40.5 =TzA I, 242.3-244.5; Amd. I, 40.5-6=TzA I, 244-5; Amd. I, 41.2=TzA I, 246-7; $A m d$. I, 59.4-5=TzA I, 330; $A m d$. I, 60.7 = TzA I, 337-8; $A m d$. I, 79.5=TzA II 401; $A m d$. I, 81.1-2=TzA II, 407; Amd. I, I I 5.I $=T z A$ II, 517; $A m d$. I, I21.4 $=T z A$ II, 539; $A m d$. I, 176.10-177.I $=T z A$ III, 734-5; $A m d$. I, I77.3 = TzA III, 736-7; Amd. I, I $89.7=T z A$ III, 785-6 (einige Stellen könnte auch als Umstandssätze zu verstehen sein).

${ }^{100}$ Amd. I, 189.2-3 = TzA III, 782-3 (vielleicht auch optativisch).
} 
$n$ sdm.n.f (präsentisch, v.a. in der Nuance 'nicht können'). ${ }^{\text {I0I }}$ Die Schreibung der Negation ist ganz überwiegend $n$, nur in einigen (meist jüngeren) Textzeugen findet man daneben auch die Variante $n n$.

Für die Frage nach altägyptischem Sprachgebrauch ist nur die Form im Wunschsatz von Bedeutung: $n s \underline{d} m . f$ in optativischer Bedeutung könnte als $n+s \underline{d} m(w) \cdot f$ (Futur/ Prospektiv) verstanden werden, oder als $n(n)+$ Subjunktiv. Im ersten Fall hätte man eine altägyptische Verbalform (die allerdings auch nach dem Alten Reich noch vorkommt), im zweiten eine alte Schreibung der Negation und die 'normale' mittelägyptische Form im negierten Wunschsatz. Die letztere Alternative ist vielleicht die wahrscheinlichere: dann hätte man auch hier eine äußerlich 'ältere' Form $(n)$ in einer jüngeren Konstruktion. ${ }^{102}$ Allerdings gibt es auch zumindest zwei Fälle, in denen tatsächlich ausgeschriebene $s \underline{d} m w$-Formen vorzuliegen scheinen. ${ }^{103}$

Im Amduat ist - wie in den anderen Unterweltsbüchern - die Konjunktion jwt ('dass nicht') nicht selten, ${ }^{104}$ die der Umwandlung negierter Hauptsätze in substantivische Nebensätze dient. Außerhalb der Unterweltsbücher kommt jwt nur im Alten Reich vor, ${ }^{105}$ im Mittelägyptischen ist es durch $n t t$ oder $w n t+$ Negation ersetzt worden. ${ }^{106}$

In Texten des Alten Reiches wird jwt hauptsächlich zur Nominalisierung von $n$ wnt 'es gibt nicht' und $n z p+$ Subjunktiv verwendet. ${ }^{107} \mathrm{Im}$ Amduat (und den anderen Unterweltsbüchern) finden sich Phrasen (dass-Sätze) mit jwt dagegen überwiegend als (nominale!) ${ }^{108}$ stichwortartige Beschreibungen und Bildbeischriften, parallel zu Infinitiven ${ }^{109}$ und Sätzen mit substantivischem $s \underline{d} m . f(m r r . f) .{ }^{100}$ Besonders häufig wird (nominales!) jwt šm.n.f $r$ st $n b(t)$ 'dass er nicht weggehen kann zu irgendeinem Ort' (u.ä.) parallel zu der für die Unterweltsbücher typischen Nominalphrase wnn.f $m$ shr $p n$ (u.ä.) 'dass er in dieser Art ist' gebraucht. ${ }^{\text {III }}$ In der Sprache der Unterweltsbücher scheinen $j w t$ und das adjektivische $j w t j$ das Negationsverb $t m$ (das in der Sprache des Alten Reiches dagegen recht häufig ist) weitgehend zu ersetzen. ${ }^{112}$

Auch hier trifft man also wieder auf Morpheme, die an sich typisch für das Alte Reich sind, in den Unterweltsbüchern aber anders verwendet werden.

ror $A m d$. I, 47.3-4=TzA I, 282-3; Amd. I, 64.5 = TzA II, 35I; $A m d$. I, 77.1 = TzA II, 392; $A m d$. I, 9I.I-2= $T z A$ II, 437; $A m d$. I, 95.10-I I $=T z A$ II, $452-3 ; A m d$. I, I27.5 $=T z A$ II, 560; $A m d$. I, I34.7 $=T z A$ II, $583(n$ vord.n.f ?); $A m d$. I, I63.I I = TzA III, 685 .

${ }_{102}$ Vgl. oben unter C) zur Schreibung und Stellung der Kopula.

${ }_{103}$ Amd. I, 60.7 $=T z A$ I, 337: $n$ hsw.tn , und $A m d$. I, I76.10=TzA III, 735: $n h p w . s n$.

104 Und ebenso die adjektivische Nominalisierung mit $j w t j$, die aber auch im Mittelägyptischen nicht selten ist.

${ }_{105} \mathrm{E} A G, \S \S 1027,1043 ; \mathrm{H}$. Satzinger, Die negativen Konstruktionen des Alt-und Mittelägyptischen (MÄS 12; Berlin, I968), $\$ \S 99-100$.

${ }_{106}$ Gardiner, $E G^{3}, \S_{1} 87$; Satzinger, Die negativen Konstruktionen, § IоI

$107 \mathrm{E} A G, \S$ ro27; Satzinger, Die negativen Konstruktionen, $\$ \$ 99-100$.

${ }^{108}$ Zur gängigen Übersetzung 'ohne dass', d.h. als Einleitung eines adverbialen Nebensatzes, s. W. Barta, 'Subjunktivische Konjunktionen als Einleitung von Umstandssätzen', GM 90 (1986), 7-10. Diese Auffassung ist m.E. unfundiert, s. Jansen-Winkeln, $S A K_{32}, 2$ I I

${ }^{109}$ Amd. I, 62.4-6=TzA II, 343-4; Amd. I, 76.6-7= TzA II, 389-90; Amd. I, 98.5-6=TzA II, 460-2.

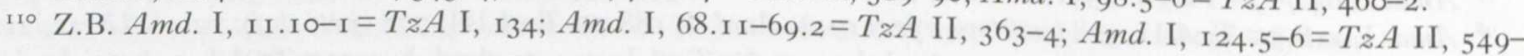
50 .

III Amd. I, 66.3=TzA II, 355-6; Amd. I, 66.8=TzA II,357; $A m d$. I, 67.9-10=TzA II, 360-1; $A m d$. I, 73.8-9 $=T z A$ II, 378-9; $A m d$. I, 8I.4 $=T z A$ II, 407-8; $A m d$. I, I I0.10-III.I $=T z A$ II, 503-4; $A m d$. I, I 52. I-3 $=T z A$ II, 640-2; $A m d$. I, 180.7-8=TzA III,750-1; $A m d$. I, 183.10-2 = TzA III, 760-2; $A m d$. I, I99.I I-2 = TzA III, 826-7; ähnlich $A m d$. I, 94.10-95. I = TzA II, 449; vgl. allgemein Jansen-Winkeln, $S A K K_{32,} 2$ 10-17, bes. $214-16$.

${ }^{112}$ Vgl. auch Jansen-Winkeln, $S A K_{32},{ }_{2}{ }_{3}$. 


\section{F) Partikeln}

Die enklitische Partikel 3 ist in einer Passage zweimal nach prädikativem Adjektiv $+w j$ belegt, wie das im Altägyptischen 'häufig' ${ }^{113}$ ist:

$n f r$ wj 3 m3s jmntjw

htp wj 3 sdm dwostjw

Re htp.f m jmnt

'wie schön ist es doch, dass die Westlichen sehen,

wie befriedigend ist es doch, dass die Unterweltlichen hören,

den Sonnengott, wenn er im Westen untergeht'. ${ }^{14}$

Nach dem Alten Reich ist 3 in diesem Kontext sehr viel seltener. ${ }^{115}$

Dagegen findet sich die nichtenklitische Partikel jst (statt $s \underline{t}$ ), die einmal im Amduat bezeugt ist, ${ }^{116}$ erst im Mittelägyptischen. ${ }^{17}$

Der 'Verstärker' $(j) r=$ ist mehrfach mit Suffix $\underline{t} n$ belegt. In drei Fällen kongruiert er (wie im Altägyptischen üblich) ${ }^{118}$ mit dem Subjekt:

$r h(w) j r . t n$

'steht (doch) auf!' ${ }^{119}$

che.tn r.t. $n$ n Repn shtj

'möget ihr euch (doch) erheben vor diesem horizontischen Re!' 20

$\underline{t} n$ r.t. $n$ r.j

'ihr gehört (doch) zu mir'. ${ }^{121}$

einmal aber auch nicht:

$w \underline{d} .[n] . j \underline{t} n$ r.t. $n$ n $n \underline{h} 3 t . j$

'ich habe euch meinem Leichnam anbefohlen'. ${ }^{122}$

Auch der Ausdruck šs m` (zur Kennzeichnung der Wirksamkeit von Sprüchen), im Amduat nur in den ersten drei Stunden verwendet, ${ }^{123}$ scheint aus dem Alten Reich noch nicht bekannt zu sein. ${ }^{124}$

\section{G) Verbalformen}

Die meisten der im Amduat gebrauchten Verbalformen kommen sowohl im Altägyptischen wie im Mittelägyptischen vor und sind daher für Datierungsfragen nicht von Belang.

${ }^{113} \mathrm{E} A G$ §834; vgl. auch Gardiner, $E G^{3}, \S \mathrm{r} 84$ n. ra; R. Weill, 'Formule énigmatique dans un texte religieux du Nouvel Empire', BIFAO $_{32}$ (1932), 60.

${ }^{114}$ Amd. I, 57.4-5 = TzA I, 317-9. Diese Phrase ist grammatisch auch dadurch bemerkenswert, dass in ihr zwei parallele Phrasen ein gemeinsames (beiden nachgestelltes) Objekt haben.

115 Z.B. $C T$ VI, $4 \mathrm{I} 3 \mathrm{~d}$.

${ }^{116}$ Amd. I, 60.3 = TzA I, 335: jst sh n sh.k Wsjr.

$117 \mathrm{~S}$. $\mathrm{E} A G \S \$_{829}, 852$ Ende.

I1 8 Vl. E $A G, \S 82 \mathrm{I}$.

${ }^{119} A m d$. I, I I4.9 = TzA II, 5 I6.

${ }^{120} A m d . \mathrm{I}, \mathrm{I} 29 . \mathrm{I}=T z A$ II, 566.

${ }^{121} A m d$. I, I29.4 $=T z A$ II, 567 . Dieser Beleg ist natürlich problematisch, da hier das $r . t n$ vermutlich durch das satzeinleitende $\underline{t} n$ bedingt ist, und diese Konstruktion ist weder 'altägyptisch' noch entspricht sie dem üblichen Mittelägyptisch.

${ }^{122}$ Amd. I, 18.5-6=TzA I, I 52-3.

${ }_{123}$ Amd. I, 22.3 = TzA I, I7I $; A m d$. I, 25. I $=T z A \mathrm{I}, \mathrm{I} 8 \mathrm{I}-2 ; A m d . \mathrm{I}, 43.8=T z A \mathrm{I}, 268 ; A m d . \mathrm{I}, 45-6[11]=T z A \mathrm{I}$, 277; $A m d$. III, 2.6=TzA I, 6; $A m d$. III, 4.7 = TzA I, r6; $A m d$. III, 4.10=TzA I, r 8; $A m d$. III, 6.8 = TzA I, 26.

${ }_{124}$ Das könnte natürlich an der Art der Textüberlieferung liegen. 
Ein altägyptisches Merkmal könnte man in dem oben $(\S \mathrm{E})$ erwähnten negierten Prospektiv $s \underline{d} m(w) . f$ sehen. Das prospektive $s \underline{d} m w . f$ kommt scheinbar noch zweimal in konsekutiver Bedeutung vor:

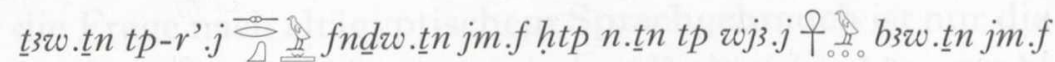

'eure Atemluft ist mein Ausspruch, so dass ihr eure Nasen von ihm atmen, euch gehört das Opfer auf meiner Barke, so dass eure Bas davon leben' ${ }^{25}$

Aber hier wird etwas nicht in Ordnung sein, denn die (Plene-)Schreibung mit w findet sich sonst eben nicht bei dreiradikaligen Stämmen, und auch das Pluraldeterminativ bei enhw ist mehr als verdächtig.

Der oft als altertümlich angesehene Komplementsinfinitiv auf .t liegt an folgenden Stellen vor:

enh.k $e_{n h t}$...

'mögest du leben ein Leben ...'.' 26

‘nh n.k ‘nh.k ‘nht b3 n.k b3.k b3t $m$ t3

'Leben sei dir, mögest du leben ein Leben, Seelenkraft sei dir, mögest du seelenhaft beseelt (o.ä.) sein in der Erde'. ${ }^{127}$

vgl. auch $h p r . k h p r t$

'du durchläufst die Verwandlungen'. ${ }^{\text {I28 }}$

Allerdings ist der Komplementsinfinitiv ${ }^{129}$ nicht auf Texte und Sprache des Alten Reichs beschränkt, er findet sich auch später noch, z.B. in den Sargtexten und in literarischen und medizinischen Texten des Mittleren Reichs. ${ }^{130}$

In den Unterweltsbüchern und auch im Amduat wird nur ein sehr begrenzter Bestand von Verbalformen gebraucht, wie verschiedentlich registriert worden ist. ${ }^{13 \mathrm{I}} \mathrm{J}$. Quack hat (für das Pfortenbuch) aus der Tatsache, dass die Konstruktion Nominalphrase $+h r$ $s \underline{d} m$ dort nicht belegt ist, auf eine Entstehung des Textes im Alten Reich oder allenfalls in der früheren I. Zwischenzeit geschlossen. Als Begründung führt er an, Vernus habe gezeigt, dass es im 'früheren Mittelägyptisch eine gut definierte Opposition zwischen dem punktuellen $\mathrm{N}+s \underline{d} m$. f und dem durativen $\mathrm{N} h \underline{h} r \underline{d} \underline{d} m$ ' gebe, 'die im späteren Mittleren Reich durch Ausweitung der letzteren Bildung aufgelöst wird'. ${ }^{{ }^{2} 2}$ Da NP $h r s \underline{d m}$ im Amduat gleichfalls nicht bezeugt ist, würde ein solcher Schluss auch hier zutreffen. Der zitierte Aufsatz von P. Vernus ${ }^{133}$ erlaubt diese Schlussfolgerung indes keineswegs. Für Vernus ist NP $h r s \underline{d} m$ (einer der) Vertreter des 'inaccompli extensif' und steht daher in Opposition (u.a.) zu NP $+s \underline{d} m . f$ als (einem der) Vertreter des inaccompli

${ }_{125}$ Amd. I, $182.5-6=T z A$ III, 756-7.

${ }_{126}$ Amd. I, 204.3 $=T z A$ III, $844^{-5}$.

${ }^{127}$ Amd. I, 119.10= TzA II 533-4.

${ }^{128}$ Amd. I, 57.7 = TzA I, 320 .

${ }^{129}$ Von Gardiner, $E G^{3}$, $\$ 298$ Obs., als einfaches Substantiv behandelt.

${ }^{13} \circ$ S. dazu P. Vernus, 'Le nom d'action et étymologique comme modificateur du verbe: Une construction égyptienne proche du maf 'ūl muțlaq', in J. C. Autuori und A. J. Quevedo Álvarez (eds.), ... Ir a buscar leña: Estudios dedicados al Prof. Fesùs López (Aula Aegyptiaca Studia 2; Barcelona, 2001), 193-202.

${ }^{131}$ J. Zeidler, Pfortenbuchstudien, I, I 35 ff. und I $89 \mathrm{ff}$.; W. Barta, Komparative Untersuchungen zu vier Unterveltsbüchern (MÄU ז; München, 1990), 126-8.

${ }^{132}$ Quack, BiOr 57, 548.

${ }^{1} 33$ P. Vernus, 'La date du Paysan Eloquent', in S. Israelit-Groll (ed.), Studies in Egyptology Presented to Miriam Lichtheim (Jerusalem, I990), II, I033-47. 
non extensif'. Diese Opposition sieht Vernus vom Alten Reich bis in die frühere I2. Dynastie in Kraft. ${ }^{134}$ Im Neuen Reich vertritt das 'Präsens I' mit Prädikat $h r+$ Infinitiv dagegen zugleich 'inaccompli extensif' und 'inaccompli non extensif'. Irgendwann zwischen I 2. und r 8. Dynastie sollte daher das Syntagma mit $h r+$ Infinitif auch in die Domäne des 'inaccompli non extensif' eingedrungen sein. Die ersten Beispiele dafür erkennt Vernus in Texten der zweiten Hälfte der I 2. Dynastie und ebenso im 'Beredten Bauern', den er daher in eben diese Zeit datiert. Aber dies sind erst Ansätze zu einem Wandel: 'soulignons que dans le Paysan éloquent ... la construction usuelle pour exprimer l'inaccompli non extensif est Sujet $+s \underline{d} m . f^{\prime} .^{135}$ In den Unterweltsbüchern und im Amduat findet man häufig NP $+s \underline{d} m . f$, und die Bedeutung eines 'inaccompli non extensif' würde dort gut passen, da diese Beischriften sich auf Bilder bzw. auf die ganze Stunde beziehen und die Vorgänge in wenigen Sätzen zuammenfassen: es handelt sich Erläuterungen, nicht um Erzählungen. Aus der Tatsache, dass in ihnen die Konstruktion NP $+s \underline{d} m . f$ nicht durch NP $h r s \underline{d} m$ ersetzt wird, folgt allenfalls, dass sie nicht in Neuägyptisch verfasst sind. Vernus hatte keineswegs behauptet, $\mathrm{NP}+s \underline{d m} . f$ sei schon in der späteren I2. Dynastie in allen Texten und Textarten durch NP $h r s \underline{d} m$ ersetzt worden.

Man kann aber auch fragen, ob das weitgehende Fehlen des Syntagmas NP $h r s \underline{d} m$ in den Unterweltsbüchern nicht zusätzlich durch die Art dieser Texte bedingt ist. Im Alten Reich wird diese Konstruktion fast nur in zitierten wörtliche Reden und in Briefen ${ }^{136}$ sowie in erzählenden Passagen (eingeleitet von $w n=$ bzw. wn.jn ${ }^{137}$ gebraucht, es scheint also noch der (nicht gehobenen) Umgangssprache anzugehören. ${ }^{1{ }^{8}}$ Auch im Mittelägyptischen ist es gerade in erzählenden Texten und Passagen besonders häufig. Es wäre also durchaus denkbar, dass der Grund für die Meidung von NP hr $s \underline{d} m$ in den Unterweltsbüchern eher in der Pragmatik zu suchen ist (in der 'Wertung' dieser Konstruktion als Merkmal einer niedrigeren Stilschicht) als in Aspekt oder Aktionsart.

Die passivische Konstruktion $j w s \underline{d}$ m.tw kommt im Alten Reich noch nicht vor, ${ }^{139}$ aber im Amduat ist sie nicht selten. Regelmäßig ist sie sogar in den 'Anbringungsvermerken' der (horizontalen) Einleitungen der einzelnen Stunden (bzw. in der Kurzfassung): $j w$ jrj.tw $n n m j$ sšm $p n$ 'dies wird gemacht entsprechend dieser Vorlage' u.ä., ${ }^{140}$ aber

${ }^{134}$ Ibid., 1036-7.

135 Ibid., I04I.

${ }^{136} \mathrm{E} A G \S 9926-30$.

${ }^{137}$ Ibid., $\$ \S 93 \mathrm{I}-2$.

${ }^{13} 8$ Erzählende Texte stehen im allgemeinen der zwanglos gesprochenen Sprache näher als Rezitationen in Kult und Ritual.

${ }^{139} \mathrm{E} A G, \S 88 \mathrm{I}$, Anm, und $\S 89 \mathrm{I}$.

140 'Anbringungsvermerke' mit jw jrj.tw: $A m d$. I, г2.2=TzA I, I35-6; $A m d . \mathrm{I}, 22.2=T z A$ I, I70; $A m d$. I, 24.8-9=TzA I, г80-1; Amd. I, 43.6=TzA I, 267; Amd. I, 45.7-8=TzA I, 275-6; Amd. I, 60.10=TzA I, 339; Amd. I, 63.6 = TzA II, 348; Amd. I, 76.8=TzA II, 391; $A m d$. I, 99.I $=T z A$ II, 463; $A m d$. I, II $8.9=T z A$ II, 529-30; $A m d$. I, 135.5 = TzA II, 585; $A m d$. I, I 54.3 = TzA III, 650-1; $A m d$. I, r68.3 $=T z A$ III, 701-2; $A m d$. I, I 80.3-4 = Tz $A$ III, 748-9; $A m d$. I, I 93.6-7 = TzA III, 798. Entsprechend in der Kurzfassung: $A m d$. III, 4.4-5 = $T z A$ I, I4-5; $A m d$. III, 6.5 = TzA I, 25; $A m d$. III, 10.3 = TzA I, 45; $A m d$. III, I 2.4 $=T z A$ I, $56-7 ; A m d$. III, I 5.8 $=T z A$ I, 68; $A m d$. III, I8.7 $=T z A$ I, 76; $A m d$. III, 20.2 = TzA I, 79-80; $A m d$. III, 22.I $=T z A$ I, 85; $A m d$. III, 23.6 = TzA I, 89; $A m d$. III, 25.3 = TzA I, 93; $A m d$. III, 26.I = TzA I, 95. Vermerke mit anderen Verben: $A m d$. I, 77.I = TzA II, 392: jw dbn.tw n.sn tp t3; Amd. I, 99.4 = TzA I, 464: jw m3 .tw n.f 3bjt.f nb $m$ ts 'geopfert wird ihm alles, was er wünscht, in der Erde'; in der Kurzfassung: $A m d$. III, го.8 $=T z A$ I, 47-8: jw wdn.tw $n n n(n) n t r w$ tp t3 'man opfert diesen Göttern auf Erden'; $A m d$. III, I $2.8=T z A$ I, $5^{8-9}$. 
sie kommt auch in den eigentlichen Stundentexten vor, ${ }^{14 \mathrm{I}}$ v.a. in der 8 . Stunde in der Phrase jw s $\underline{d}$ m.tw hrw jht m qrrt tn 'man hört die Stimme von etwas aus dieser Höhle', ${ }^{142}$ ähnlich in der 5. Stunde: jw sd m.tw hrw jht $m$ nwt tn 'man hört die Stimme von etwas aus diesem Oval'. ${ }^{43}$

\section{H) Kongruenz im Spaltsatz}

Ein charakteristischer Unterschied zwischen Alt- und Mittelägyptisch ist die Kongruenz im Spaltsatz: im Altägyptischen kongruiert das Partizip in Genus und Numerus, ${ }^{\text {I44 }}$ im Mittelägyptischen bleibt es unverändert (ohne Genus- und Numerusendung). ${ }^{\text {I45 }}$ Im Amduat wird diese Kongruenz sehr merkwürdig gehandhabt:

a) mit nominalem Subjekt:

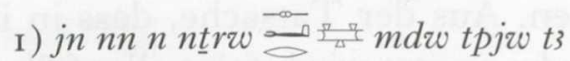

'es sind diese Götter, die aufsteigen lassen die Worte derer, die auf Erden sind'. ${ }^{\text {I46 }}$

2) jn nn n wjjzw št $3 w-\infty$

'es sind diese unzugänglichen Barken, die ihn leiten in diesem Feld'. ${ }^{147}$

3) $j n n s(r) w \operatorname{tpj}(w) r$ ' $n w j 3 . f^{-\infty} \wedge s w m n n n w 3 w t \check{s} t 3 w t$

'es sind die Flammen, die auf dem Maul seiner Barke sind, die ihn leiten auf diesen unzugänglichen Wegen'. ${ }^{148}$

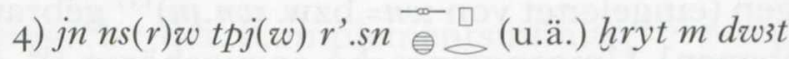

'es sind die Flammen, die auf ihrem Maul sind, die das Abschlachten (der Feinde) verursachen in der Unterwelt'. ${ }^{\text {I49 }}$

5) jn nswt tpj(w)t r'n jerwt.sn

'es sind die Flammen, die auf dem Maul ihrer Uräen sind, die Apophis von Re abwehren'. ${ }^{150}$

b) mit Pronomen der 3. Pl. (ntsn):

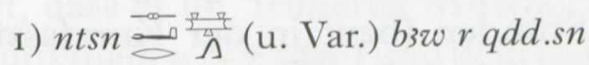

'sie sind es, die die Seelen zu ihrem Schlaf gelangen lassen'. ${ }^{151}$

${ }^{14 \mathrm{I}}$ Amd. I, I 23.2 = TzA II, 545: jw jrj.tw nn $n$ ḩkзw 'man macht diese Zauber ...'; $A m d$. I, I 23.4 = TzA II, 546: jw jrj.tw tp t3 $m$ mjtt 'man macht (es) auf Erden ebenso'.

${ }_{\mathrm{I} 42} A m d$. I, 136.4 = TzA II, 589; Amd. I, 137.4=TzA II, 592-3; Amd. I, ${ }_{3} 8.3=T z A$ II, $596 ; A m d$. I, I39.5 = $T z A$ II, 599; $A m d$. I, 140.6=TzA II, 603; $A m d$. I, 148.2 = TzA II, 628; $A m d . \mathrm{I}, \mathrm{1} 49 \cdot 3=T z A$ II, 63I; $A m d$. I, I $50.2=T z A$ II,634; $A m d$. I, I $51.4=T z A$ II, 638;Amd. I, I 52.4 = TzA II, 642 .

${ }^{143}$ Amd. I, 93.6 = TzA II, 444 .

${ }^{144}$ K. Sethe, Das ägyptische Verbum im Altägyptischen, Neuägyptischen und Koptischen (Leipzig, I9I3), II, $\S \S 752-3$; $\mathrm{E} A G \S \S 632,945$, mit weiteren Belegen bei E. Doret, 'Phrase nominale, identité et substitution dans les textes des sarcophages (première partie)', $R d E 40$ (I989), 50, n. I2; J. Allen, The Inflection of the Verb in the Pyramid Texts (Malibu, I984), §408; E. Doret, 'Cleft-sentence substitutions et contraintes sémantiques en égyptien de la première phase (V-XVIII Dynastie)', LingAeg I (r99I), 58 .

${ }_{145}$ Gardiner, $E G^{3}, \S 373$. In den Sargtexten findet sich teilweise noch Kongruenz, s. Doret, $\operatorname{Ling} A \operatorname{eg}$ I, 58 ; id., $R d E_{4}$ ०, $50 \mathrm{nn} .8-9$.

${ }_{146}$ Amd. I, 25.8=TzA I, 184 .

${ }^{147}$ Amd. I, 50.7 $=T z A \mathrm{I}, 293-4$.

${ }_{148}$ Amd. I, 69.1 $=T z A$ II, 364 .

${ }_{149}$ Amd. I, $163.8=T z A$ III, 683-4.

${ }_{150}$ Amd. I, 194.2 = TzA III, 802 .

${ }^{151}$ Amd. I, 25.8-26. I $=T z A \mathrm{I}, \mathrm{1} 84-5$. 


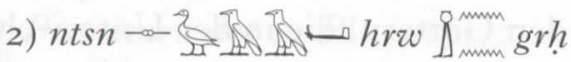

'sie sind es, die den Tag hüten und die Nacht bringen'. ${ }^{152}$

3) $n t s n{ }_{\text {mus }}^{-\infty} \underline{d} \underline{d} t \ldots n n \underline{t} r w$

'sie sind es, die den Göttern die Pflanzen ... zu essen geben'. ${ }^{153}$

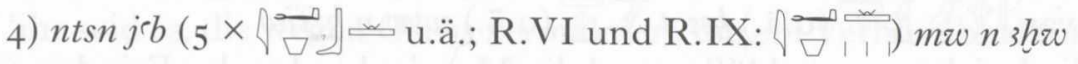
'sie sind es, die den Verklärten Wasser geben'. ${ }^{154}$

5) ntsn Ð8 A

'sie sind es, die die Glut erhitzen, um die Feinde des Re zu verbrennen'. ${ }^{55}$

6) $n t s n$ I $h 3 t j w h r s d t$

'sie sind es, die die Herzen auf die Flamme werfen'. ${ }^{156}$

7) $n t s n=-n h p$

'sie sind es, die den "Beschützer" hüten'. ${ }^{157}$

8) $n t s n ?-7 n p w$

'sie sind es, die Anubis hüten'. ${ }^{158}$

9) $n t s n$ 罗

'sie sind es, die beim Bestrafen der Toten stehen in der Unterwelt'. ${ }^{159}$

го) $n t s n$ (fem.) $\stackrel{-\infty}{2} \Delta$ (u.ä.) $e^{-\infty}$ w wss

'sie sind es, die Leben und Heil aufsteigen lassen'. ${ }^{160}$

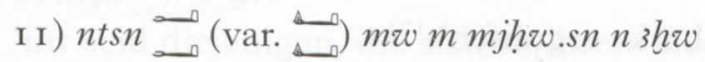

'sie sind es, die den Verklärten Wasser geben mit ihren Rudern'. ${ }^{161}$

1 2) $n t s n \stackrel{-\infty}{\curvearrowright}$ b3 $m$ jrw.f

'sie sind es, die den Ba in seinen Gestalten erscheinen lassen'. ${ }^{162}$

13) $n t s n-$ (var. $\stackrel{\leftrightarrow}{\hookrightarrow})$ 3wt n ntrw jmjw dwst

'sie sind es, die Opfer geben den Göttern, die in der Unterwelt sind'. ${ }^{163}$

I4) $n t s n$ (fem.) 192 o kkw $m$ `t h hrjt Wsjr

'sie sind es, die die Finsternis erleuchten in dem Raum, in dem sich Osiris befindet'. ${ }^{164}$

I 5) $n t s n \stackrel{\theta}{2} h f_{3} w t$ nbt jmjt t3

'sie sind es, die jede Schlange abwehren, die in der Erde ist'. ${ }^{165}$

I6) $n t s n+$ (u.Var.: $6 \times$ ohne Pluraldet; Th III: $\left.{ }^{-\infty}\right) m d w n n t r w j m j w d w s t$

${ }^{152}$ Amd. I, 26.3 = TzA I, $185-6$.

${ }^{153}$ Amd. I, 33.6 = TzA I, 208.

${ }^{154}$ Amd. I, 33.8 = TzA I, 208-9.

${ }_{155}$ Amd. I, 34.I $=T z A \mathrm{I}, 209-10$.

${ }^{156}$ Amd. I, 34.2 = TzA I, 210 .

${ }_{157}$ Amd. I, 68.3 = TzA II, 362 .

${ }_{15}{ }^{8} A m d$. I, 7I.I $=T z A$ II, $37 \mathrm{I}$.

${ }^{159}$ Amd. I, 82.7 = TzA II, 4I I-2.

${ }^{160}$ Amd. I, I 57.7 $=T z A$ III, 664 .

${ }_{161}$ Amd. I, 160.5 $=T z A$ III, 673 .

${ }^{162}$ Amd. I, 160.7 = TzA III, 673-4.

${ }^{163} A m d$. I, 161.1-162.1 I $=T z A$ III, 678.

${ }_{164}$ Amd. I, 163.7 $=T z A$ III, $68_{3}$.

${ }^{165} A m d$. I, 163.9 $=T z A$ III, 684 . 
'sie sind es, die Stäbe (zur Speise?) darreichen den Göttern, die in der Unterwelt sind'. ${ }^{166}$

17) $n t s n \stackrel{-\infty}{-} h t n b$ rdyt nbt $n$ nwt tn

'sie sind es, die alle Bäume und alle Pflanzen entstehen lassen für diese Stätte'. ${ }^{167}$

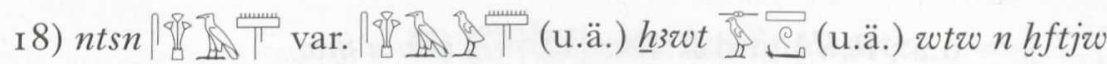

'sie sind es, die die Leichname entblößen und die Mumienbinden der Feinde zusammenballen'. ${ }^{\text {68 }}$

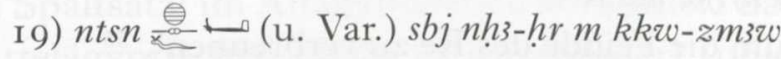

'sie sind es, die den Widersacher Nh3- hr in der Urfinsternis abwehren'. ${ }^{169}$

20) $n t s n$ (fem.) $199^{2}$ ○) wst $n$ Re $m$ kkw-zmsw

'sie sind es, die den Weg des Re in der Urfinsternis erleuchten'. ${ }^{170}$

2i) $n t s n$ (fem.)

'sie sind es, die das heilige Tor von Sais hüten'. ${ }^{171}$

22) ntsn (fem.)

'sie sind es, die die "Rötung" machen (das Blutgericht abhalten) mit den Feinden des Osiris in der Unterwelt'. ${ }^{172}$

23) $n t s n$ (fem.) 1$\}$ ? (u.ä.) jb n ntrw jmnt

'sie sind es, die das Herz der Götter des Westens erfreuen'. ${ }^{173}$

24) $n t s n$ (fem.) $n f r t n t w j 3 n R e$

'sie sind es, die das Zugseil der Sonnenbarke ergreifen'. ${ }^{174}$

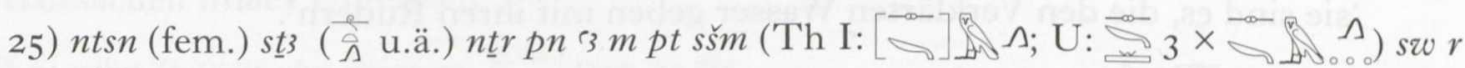
wswt hrt

'sie sind es, die diesen großen Gott im Himmel ziehen, die ihn zu den Wegen des Himmels geleiten'. ${ }^{175}$

26) $n t s n$ (fem.) $\stackrel{\text { \& }}{\diamond}$ prt $m p t$

'sie sind es, die das machen, was am Himmel geschieht'. ${ }^{176}$

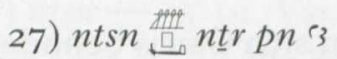

'sie sind es, die diesen großen Gott empfangen'. ${ }^{177}$

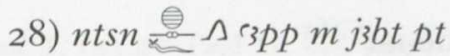

'sie sind es, die Apophis im Osten des Himmels abwehren'. ${ }^{178}$

${ }^{166}$ Amd. I, $165 \cdot 7=T z A$ III, $69 \mathrm{I}-2$.

${ }_{167} A m d . \mathrm{I}, \mathrm{I} 65.8=T z A$ III, 692.

${ }_{168}$ Amd. I, I71.9 = TzA III,715-6.

${ }_{169}$ Amd. I, I 75.4 = TzA III, 728-9.

${ }_{170}$ Amd. I, I78.3 = TzA III, 741.

${ }^{171}$ Amd. I, 188.2 = TzA III, 778 .

${ }_{172}$ Amd. I, I91.3 = TzA III, 789-90.

${ }_{173} A m d$. I, 194.6 $=T z A$ III, 805 .

${ }_{174}$ Amd. I, 200.7 = TzA III, 829 .

${ }_{175}$ Amd. I, 200.8-9 $=T z A$ III, 830.

${ }_{176} A m d$. I, 200.10 = TzA III, 830-I.

${ }_{177}$ Amd. I, 202.4 $=T z A$ III, 835 .

${ }_{178} A m d . \mathrm{I}, 202 . \mathrm{I}$ I $=T z A$ III, 837 . 
c) mit Pronomen der 2. Pl. (nttin):

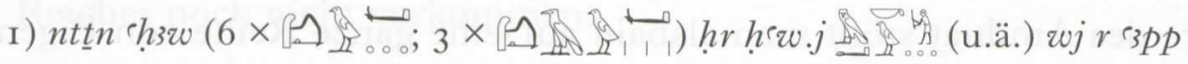

'ihr seid es, die für meinen Leib kämpfen, die mich schützen gegen Apophis'. ${ }^{179}$

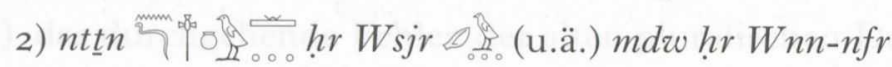

'ihr seid es, die wegen Osiris zermalmen, die ein Verhör abhalten wegen Onnophris'. ${ }^{80}$

3) $n t \underline{t} n$ 參

'ihr seid es, die auf Erden entstanden sind und die jubeln über das Vertreiben meiner Feinde'. ${ }^{18 \mathrm{I}}$

4) $n t \underline{t} n \stackrel{\theta}{\Delta} m h t p w$

'ihr seid es, die zufrieden sind mit den Opfern'. ${ }^{82}$

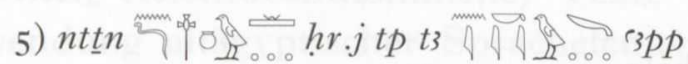

'ihr seid es, die mir huldigen auf Erden, die den Apophis bestrafen'. ${ }^{183}$

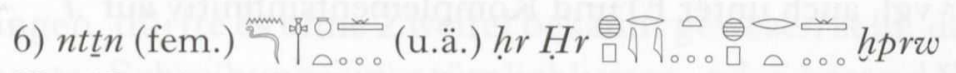

'ihr (Göttinnen) seid es, die Horus huldigen, die entstanden sind und das (zu)

Entstehende entstehen lassen'. ${ }^{184}$

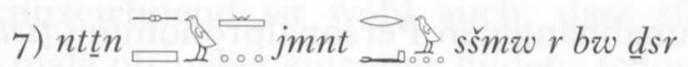

'ihr seid es, die das Verborgene öffnen und das Bild an seinen abgeschiedenen Ort setzen'. ${ }^{185}$

8) $n t \underline{t} n \stackrel{\theta}{\rightarrow \square} h r t$

'ihr seid diejenigen, die sich an ihrem Bedarf befriedigen' (etwas unsicher). ${ }^{186}$

Mit einem Subjekt in der 3. Person findet sich also in Genus ${ }^{187}$ und Numerus durchgehend keine Kongruenz (im Numerus mit ganz wenigen, wohl z.T. scheinbaren oder irrtümlichen Ausnahmen). ${ }^{188}$ Steht das Subjekt dagegen in der 2. Person, kongruiert das Partizip immer in Genus ${ }^{189}$ und Numerus.

Eine Erklärung für diese Erscheinung fällt schwer. Möglicherweise wusste der Verfasser bzw. Redaktor, dass in alter Zeit das Partizip kongruierte, aber nicht genau, unter welchen Umständen.

${ }^{179}$ Amd. I, $42 \cdot 3=T z A$ I, 256.

${ }^{180}$ Amd. I, 82.3 $=T z A$ II, 410 .

${ }^{181}$ Amd. I, 102.3 = TzA II, 474 .

${ }^{182}$ Amd. I, rog.8 = TzA II, 498 .

${ }^{183}$ Amd. I, ${ }_{109.9}=T z A$ II, $498-9$.

${ }^{18_{4}}$ Amd. I, 170.6 = TzA III, 710-1.

${ }^{185} A m d$. I, I82.4 = TzA III, 755-6.

${ }^{186}$ Unsicher: $A m d$. I, Iо0. I $=T z A$ II, 466.

${ }_{187}$ Ein feminines Bezugswort bei A.5 und B. 10, B. 14, B.20-26.

${ }_{188}$ In B.4 haben nur die späten Belege bei R.VI und R.IX Pluralstriche, die 5 älteren Versionen nicht; im B. I 6 hat Th.III Pluralstriche (wohl irrtümlich, da alle Wörter vorher und nachher im Plural stehen), alle anderen 6 Versionen nicht, in B.25 ist das I. Partizip ( $s \underline{t 3}$ ) überall unmarkiert (mask. Sg.), das 2. (š̌m) in den ältesten Versionen (Th.I und U) unmarkiert, in 3 anderen mit Pluralstrichen markiert. In B. 8 haben 4 Versionen sḩ, eine (R.VI) gibt sḩ3, und Th.I und U haben sḩsw: dabei dürfte es sich kaum um eine Pluralschreibung handeln, die Verben mit 3 als letztem Radikal zeigen öfter Unregelmäßigkeiten; s. E. Edel, 'Beiträge zur ägyptischen Grammatik', ZÄS 84 (1959), ro6-o8.

${ }^{189}$ Feminines Subjekt bei C.6. 


\section{I) Zusammenfassung}

Bei der Lektüre des Amduat stößt man alsbald auf eine ganze Reihe von Eigentümlichkeiten, die auf den ersten Blick der Sprache des Alten Reiches zu entsprechen scheinen:
A) bestimmte für das Alte Reich typische Schreibungen ${ }^{190}$
B) die älteren Demonstrativa $p w$ und $t w$ (neben den häufigeren $p n$ und $t n$ ) und Voran- stellung oder selbständiger Gebrauch adjektivischer Demonstrativpronomina
C) Schreibung der Kopula als $p j$ oder $p$
D) die unabhängigen Personalpronomina twwt und swt
E) die einheitliche Negation $n$ (ohne Unterscheidung von $n$ und $n n$ ) und die negative Konjunktion jwt
F) bestimmte Gebrauchsweisen von Partikeln
G) prospektives $s \underline{d} m(w) . f($ ? vgl. auch unter E) und Komplementsinfinitiv auf.$t$
H) Kongruenz im Spaltsatz

Davon kommen einige Merkmale allerdings auch noch in Texten vor, die nach dem Alten Reich entstanden sind, wie z.B. die unabhängigen Personalpronomina twt und swot (D) oder der Komplementsinfinitiv (G).

Vor allem aber ergibt sich bei näherer Betrachtung, dass ein erheblicher Teil dieser 'Altägyptizismen' nicht wirklich alt sein dürfte:

A) Einige der 'alphabetischen' Schreibungen sind von einer Art, wie sie in einem wirklich altägyptischen Text kaum vorkommen würden.

B) Die Verwendung vorangestellter und selbständiger Demonstrativpronomina ist anders als im Alten Reich.

C) Die Schreibung der Kopula als $p j$ oder $p$ ist sogar häufiger als im Alten Reich und damit etwas suspekt, ihre Stellung im Satz entspricht zudem mittelägyptischem Gebrauch.

D) Die Konstruktionen mit vorangestelltem enklitischen Pronomen ( $s w s \underline{d} \underline{d} . f)$ kommen in nachweislich alten Texten nicht vor.

E) Die negative Konjunktion jwt ist im Amduat (und in den Unterweltsbüchern insgesamt) sogar häufiger als im Alten Reich, ihre Verwendung ist allerdings nicht dieselbe wie im Altägyptischen dieser Zeit.

H) Die Kongruenz im Spaltsatz wird auf eine sehr eigentümliche Weise gehandhabt, jedenfalls nicht so wie im Altägyptischen.

Daraus ergibt sich, dass diejenigen 'Altägyptizismen', die so auch im Alten Reich vorkommen könnten, sich vorwiegend auf 'äußere' Merkmale und Formen beschränken: bestimmte Schreibungen, einige alte Formen der Demonstrativ- und Personalpronomina, einheitliche Negation, Partikel.

Wenn es aber um den Gebrauch der alten Formen geht, entspricht die Sprache des Amduat dort, wo Altägyptisch und Mittelägyptisch sich unterscheiden, nicht dem Altägyptischen: bei den eher unauffälligen Spracheigenschaften wie Wortstellung und Kongruenz zeigt sich, dass die zugrundeliegende Sprache das Mittelägyptische ist.

${ }^{190} \mathrm{Zu}$ den Belegen s.o. unter den entsprechenden Buchstaben. 
Dazu kommt, dass es außerdem auch einige Merkmale gibt, die in der Sprache des Alten Reiches noch nicht vorkommen:
A) 'hyperkorrekte' Schreibungen
B) das durchgehende Fehlen der alten pluralischen Demonstrativa (wie $j p n / j p t n$ )
C) die Stellung der Kopula
D) das Pronomen st und der Possessivausdruck $n . k$-jmjj
F) die Partikel $j s \underline{t}$
G) die Verbalform $j w s \underline{d} m . t w$

Dies alles erklärt sich am ehesten, wenn es sich nicht um wirklich alte (Vorlagen des Alten Reiches entnommene) Texte handelt, sondern um eine archaisierende Verwendung altägyptischer Sprachelemente. Zumindest den gelehrten Schreibern und 'Theologen' der Epochen nach dem Alten Reich, die ständig mit älteren Texten umgingen, dürfte es ohne Zweifel bekannt gewesen sein, dass bestimmte grammatische Elemente, Schreibungseigentümlichkeiten, oder besonders prägnante Verwendungen (wie die Voranstellung der Demonstrativa) ein Kennzeichen sehr alter Texte waren.

Kennzeichnend ist wohl auch, dass sich (scheinbar) altägyptische und jüngere Merkmale oft nebeneinander finden: schon H. Grapow hatte die alte Schreibung der Kopula als $p j$, die sich vor allem in der 4 . und 5 . Stunde findet, als Indiz dafür gewertet, dass diese beiden Abschnitte besonders alt seien. ${ }^{19 \mathrm{I}}$ Es ist aber zu bemerken, dass sich gerade in diesen Stunden auch besonders häufig 'hyperkorrekte' Schreibungen finden wie etwa die Femininendung als $\approx$.

Der Schluss liegt also nahe, dass man dem Text des Amduat ein möglichst altehrwürdiges Gepräge geben wollte, und ebenso den anderen Unterweltsbüchern und verwandten Texten wie etwa der Sonnenlitanei. Denn es ist auffallend, dass alle diese Kompositionen (z.T. zahlreiche) 'Altägyptizismen' enthalten-aber alle eine etwas andere Auswahl und in etwas anderer Verwendungsweise. ${ }^{192}$ Das wäre schwer zu erklären, wenn es sich wirklich um Werke des Alten Reiches handelte.

Man kann die Sprache des Amduat also 'archaisierend' nennen, oder, da altägyptische Sprachelemente ja in vielen religiösen Texten ganz unterschiedlicher Art vorkommen, mit W. Schenkel allgemein von einer 'Fachsprache' für religiöse Texte reden, die in größerem Umfang Elemente des Altägyptischen enthält. ${ }^{193}$ Die eigentliche Grundlage dieser Fachsprache dürfte aber das Mittelägyptische sein, wie nicht zuletzt das Amduat deutlich zeigt.

Die Tatsache, dass Sprache und Schrift des Amduat öfter 'archaisierend' ist, muss aber noch keineswegs bedeuten, dass einzelne Elemente darin nicht tatsächlich sehr alt sein könnten. Es ist immerhin sehr wahrscheinlich, dass dieser Text eine längere Redaktionsgeschichte hatte, ${ }^{194}$ und dann könnten einzelne Teile des Gesamtwerks aus Vorlagen des Alten Reiches stammen. Aufgrund der gleichförmigen Gliederung des Textes und der Formelhaftigkeit vieler seiner Einzelteile in seiner vorliegenden Gestalt

${ }^{191}$ Grapow, ZÄS 72, 34 .

${ }^{192}$ Z.B. findet sich die Voranstellung adjektivischer Demonstrativa auch häufig in der Sonnenlitanei, dort aber besonders gern mit folgendem Relativsatz: s. E. Hornung, Das Buch der Anbetung des Re im Westen, I (AH 2; Geneva, 1975), 6, 7, 9, 10, I1, I2, 13, I4, I5, 60.

${ }_{193}$ W. Schenkel, Tübinger Einführung in die klassisch-ägyptische Sprache und Schrift (Tübingen, I997), I9, 20.

${ }_{94}$ Rößler-Köhler, in Gundlach und Seipel (eds), Das frühe ägyptische Königtum, 73-4. 
ist allerdings anzunehmen, dass er durchgehend (und eingehend) redigiert worden ist. Aus ganz alter Zeit dürften dann eher nur Worte und Einzelelemente stehengeblieben $\operatorname{sein}^{195}$ als ganze Passagen. Und dass der uns vorliegende Text des Amduat insgesamt oder in wesentlichen Teilen aus dem Alten Reich stammt, wird man mit gutem Gewissen ausschließen können.

Die Datierung des Amduat und der anderen Unterweltsbücher wird vorausssichtlich auch in Zukunft noch kontrovers erörtert werden. Beruft man sich dabei auf die Sprache dieser Texte, wäre es aber empfehlenswert, nicht nur vereinzelte 'altägyptische' Formen als Hinweis oder gar Beweis für ihre zeitliche Einordnung heranzuziehen, sondern ihren Sprachgebrauch insgesamt zu untersuchen. ${ }^{195}$ Schreibungen wie $j . f($ statt $j w . f)$, das Spitzbrot als Imperativ $j m j$ oder $d w$ als Infinitiv von $w d j$ (s.o., §A)
könnten beispielsweise solche Überreste sein. 